\title{
BMJ Open Prevalence and associated factors of khat chewing among students in Ethiopia: a protocol for systematic review and meta-analysis
}

\author{
Wondale Getinet Alemu, ${ }^{1}$ Tadele Amare Zeleke, ${ }^{1}$ Wubet Worku Takele ${ }^{2}$
}

To cite: Alemu WG, Amare Zeleke T, Takele WW.

Prevalence and associated factors of khat chewing among students in Ethiopia: a protocol for systematic review and meta-analysis. BMJ Open 2018;8:e21157. doi:10.1136/ bmjopen-2017-021157

- Prepublication history for this paper is available online. To view these files, please visit the journal online (http://dx.doi org/10.1136/bmjopen-2017021157).

Received 13 December 2017 Revised 5 November 2018 Accepted 6 November 2018
Check for updates

(c) Author(s) (or their employer(s)) 2018. Re-use permitted under CC BY-NC. No commercial re-use. See rights and permissions. Published by BMJ.

${ }^{1}$ Department of Psychiatry, College of Medicine and Health Sciences, University of Gondar, Gondar, Ethiopia

${ }^{2}$ Department of Community Health Nursing, School of Nursing, College of Medicine and Health Sciences, University of Gondar, Gondar, Ethiopia

Correspondence to Wondale Getinet Alemu; wondale22@gmail.com

\section{ABSTRACT}

Introduction These days, in Ethiopia, khat chewing is one of the widely spreading public health problems affecting the most productive segment of the population. The health implications of khat chewing among students are strongly linked with poor mental, physical and social performances. However, the national magnitude of khat chewing and the associated factors among Ethiopian students are unknown. Therefore, this systematic review and meta-analysis will answer the national prevalence of khat chewing and the associated factors among students in Ethiopia. Methods Published primary relevant articles will be accessed using various databases, such as Medline, PubMed, EMBASE and Scopus. Other electronic search engines, for instance, Google Scholar and Google, will be used. Furthermore, additional studies will be collected by communicating with the author(s) and following the references of relevant articles. To select eligible studies, the Joanna Briggs Institute quality appraisal checklist will be used. The Preferred Reporting Items for Systematic Reviews and MetaAnalyses checklist will be followed to keep the scientific rigour of the study. Heterogeneity between studies will be examined through forest plot and $\mathrm{I}^{2}$ heterogeneity tests. To identify influential studies, sensitivity analysis will be done. For substantial heterogeneity $\left(I^{2}>50 \%\right)$, the DerSimonian and Laird random-effects model will be employed. Subgroup analyses will be conducted using the random-effect model. Moreover, small studies' publication bias will be checked by funnel plots and objectively by Egger's regression test. If in case Egger's test was found to be statistically significant $(p<0.05)$, trim and fill (Duval and Tweedie) analysis will be performed. The presence of association will be declared using $\mathrm{p} \leq 0.05$ and $\mathrm{OR}$ with corresponding $95 \% \mathrm{Cl}$.

Ethics and dissemination Since the intention of the study is to describe earlier primary studies qualitatively and pool the results of those articles, ethical clearance will not be a concern. The results of the study will be published in a reputable peer-reviewed journal and presented at different scientific research conferences. It will also be disseminated to academic as well as other concerned institutions.

PROSPERO registration number CRD-42,017,081,886.
Strengths and limitations of this study

The study will be the first of its kind in Ethiopia.

- The findings of this review will be highly crucial to both governmental and non-governmental organisations that are engaged in health policymaking linked to substance use, specifically the use of khat and its impact.

- A broad scope of participants and areas in sections of the society will be covered.

- Since the studies will be collected all over Ethiopia, heterogeneity among studies will be a threat, which might deter pooling of the findings.

\section{BACKGROUND}

Khat is a leafy plant with natural amphetamine content which has been predominantly planted in the East African and Arabic land. ${ }^{12}$ The name of this plant varies from country to country. Nevertheless, 'khat' is the common name generally used in scientific studies. ${ }^{3}$ The origin of khat is not clearly known, but some people presumed it is from Ethiopia. ${ }^{4}$ Khat contains the highest psychoactive chemicals, including but are not limited to cathinone and other chemicals. ${ }^{5}$

Khat chewing has remarkable stimulant effects on people's nervous system. ${ }^{67}$ To put it differently, the new fresh sheets and new buds of khat are rich in amphetamine. ${ }^{8}$ As a result, users could develop tachypnoea, hyperthermia, tachycardia, mydriasis, restlessness and relaxation. ${ }^{9}$ The drug effects are enhanced mutually with caffeine and cigarette smoking. ${ }^{10}$ Culturally, even though khat chewing is believed to be only for men, women are also known khat users. ${ }^{11}$ People typically start chewing at an earlier age and grows into an uncontrollable daily habit lasting a lifespan. ${ }^{12}$ Khat use is seen in local practices and habits in Ethiopia. ${ }^{13}$ In addition, it is becoming a great aspect of both cultural and spiritual services. ${ }^{1415}$ 
According to WHO, khat use could lead to dependency ${ }^{16-20}$ Studies reported that prolonged usage of khat exposes the user to thrombocytosis, which predisposes to myocardial infarction, ${ }^{21}$ ischaemic heart disease, ${ }^{22}$ maniclike schizophrenia ${ }^{23}{ }^{24}$ and distress secondary to withdrawal. ${ }^{25}$ Likewise, its use has multiple deleterious effects, including erectile dysfunction, ${ }^{26}$ unsafe sex, ${ }^{27}$ psychotic experience, ${ }^{28} 29$ oesophageal tumour, ${ }^{30}$ low birth weight and lactation problem, ${ }^{31}$ and anatomical as well as functional brain changes. ${ }^{32}{ }^{33}$ Moreover, behavioural impairment associated with its use might lead to a variety of criminal deeds. ${ }^{34}$

Nowadays, khat chewing is reported to be the most common malpractice among high school, college and university students. In other words, they believe that its use would keep them alive and attain greater concentration, and that it boosts pleasure, enhances motivation and strengthens socialisation. ${ }^{35-37}$

Studies in different corners of the globe revealed that this unwanted behaviour seemed to be a critical problem; for instance, in Saudi Arabia, it was reported to be between $23.1 \%$ and $30.3 \% .{ }^{38-40}$ In addition, in Ethiopia, the prevalence ranged from $6.95 \%$ to $64.9 \%$ in Oromia and Amhara regions, respectively. ${ }^{41-45}$ In earlier studies done elsewhere, multiple factors have contributed to khat chewing. Namely, the higher likelihood of khat chewing was observed among male students whose friends chew khat, alcohol drinkers and students whose families are cultivating khat in their garden. ${ }^{3546-48}$

In Ethiopia, a lot of studies have been conducted among high school, college and tertiary students. However, the findings of those studies showed inconclusive reports on the different magnitude and factors. To mitigate the magnitude, showing pooled national estimates of khat chewing and identifying the contributing factors are quite crucial. Therefore, the aim of this study will be to determine the pooled prevalence of khat chewing and the associated factors among students in Ethiopia. Findings could help in designing possible strategies to combat the magnitude of khat chewing in the country.

\section{METHODS AND ANALYSIS}

\section{Protocol registration and review reporting}

This protocol has been registered in the international prospective register of systematic reviews and meta-analysis (PROSPERO) and the PROSPERO registration number is CRD-42,017,081,886. While preparing the protocol, the Preferred Reporting Items for Systematic Reviews and Meta-Analyses (PRISMA) checklist was used to maintain the scientific rigour of the study. Likewise, the Meta-analysis Of Observational Studies in Epidemiology guidelines ${ }^{49}$ and the PRISMA reporting checklist will be used to report the findings of the study. ${ }^{50}$ Previous studies will be qualitatively described based on the region where the studies were conducted, the level of students and important factors. The screening and selection processes will be presented using PRISMA flow diagram. The findings of the study will be illustrated through figures as well as detailed text.

\section{Data source and search strategy}

Published primary studies will be gathered using PubMed, Scopus, Medline, HINARI and other databases. More articles will be retrieved using electronic search engines such as Google and Google Scholar. References of the relevant articles will be followed to access additional studies. Furthermore, for articles where there is difficulty accessing the full text and other necessary information, the corresponding author(s) will be contacted via email or other means of communication. The key search terms will be 'Khat chewing', 'determinant factors', 'associated factors', 'high school student', 'College students', 'University students' and 'Ethiopia'. Using all these terms, relevant topics will be searched in an advance search of databases through 'MeSH terms' and 'All fields' using the connecting 'AND 'and 'OR' Boolean operator terms as appropriate. All these processes will be performed from 20 October 2017 to 20 December 2017 , and the study is expected to be completed by 13 February 2019.

\section{Inclusion and exclusion criteria}

Cross-sectional, analytical cross-sectional, case-control and cohort studies will be considered. All studies written in the English language will be included. As long as studies are reporting the prevalence and the associated factors of khat chewing and are conducted in Ethiopia, they will be included without time restriction. Articles without full text and where there is difficulty extracting data will be excluded.

\section{Study screening and selection}

First, all articles accessed from databases and electronic search engines will be exported to EndNote V.6, and duplicate studies will be identified and then removed. Second, the remaining articles will be evaluated based on the topic, language and study area. Accordingly, studies conducted outside of Ethiopia, studies documented other than the English language and irrelevant topics will be rejected. Finally, abstracts and full texts of the remaining articles will be thoroughly reviewed.

\section{Patient and public involvement}

The study protocol was prepared by WGA, TAZ and WWT. The study will include previous primary studies that have been done among students of Ethiopia. Thus, individuals will not be part of this study. Three researchers (WGA, TAZ and WWT) will be involved in analyses, interpretation of the results, editing, preparing the manuscript for publication, as well as disseminating the findings to different academic and other concerned institutions. Furthermore, the study is simply aggregating and summarising of findings of earlier studies authored by different scholars, and therefore we would highly like to acknowledge them. 


\section{Risk of bias and quality assessment}

The Joanna Briggs Institute critical appraisal checklist will be used to appraise the quality of studies. ${ }^{51}$ Quality assessment will be done by three independent reviewers (WWT, WGA and TAZ). Whenever there is any inter-rater arguments between the reviewers, a detailed evaluation and investigation of the sources of the discrepancy will be performed. If the disagreements persist, the average of the three reviewers will be calculated. Once the scores are converted to percentages, only studies that scored $\geq 50 \%$ will be included in the study. For every factor, the same procedures will be followed. Moreover, for all eligible studies, the quality score will be placed in a data extraction format.

\section{Data collection process}

Once eligible studies are identified, two independent reviewers (WGA and WWT) will extract the data using a prepared format on a Microsoft Excel spreadsheet. The following are the variables which will be extracted: author(s) name, sample size, response rate of the studies, study year/year of publication, region where the study(s) was conducted, level of students (high school, college and university), religion, ethnicity and sex of the participants. For prevalence studies, the prevalence, logarithm of prevalence and SE of the logarithm of prevalence will be computed. Similarly, for factors, important variables of OR, logarithms of OR and SE of the logarithms of OR will be calculated. Moreover, for any difficulties faced during data extraction, the corresponding author will be contacted by any means of communication.

\section{Outcome variable}

Khat chewing is defined as the proportion of students who are chewing khat for various reasons.

\section{Assessment of publication bias and data analysis}

After the necessary data are extracted and documented in the Microsoft Excel spreadsheet, the data will be exported to STATA/SE V.14 for further analysis. In the first step, in the fixed-effect model, the presence of heterogeneity among studies will be examined using the $\mathrm{I}^{2}$ heterogeneity test. ${ }^{52} \mathrm{I}^{2}$ heterogeneity test of $>50 \%$ will be declared as the presence of heterogeneity. Thus, the DerSimonian and Laird random-effect model will be used. Influential studies will be identified through sensitivity analysis and necessary measures will be taken as per the result. Subgroup analyses will be performed and could be based on region, students' educational level and study year. Small studies' publication bias will be examined using a symmetric funnel plot and objectively through Egger's regression test. ${ }^{53}$ Asymmetry of the funnel plot or statistical significance of Egger's regression test $(\mathrm{p}<0.05)$ will be suggestive of publication bias. Therefore, trim and fill (Duval and Tweedie) analysis will be conducted. Using the DerSimonian and Laird random-effect model, the pooled prevalence and the OR will be reported at $95 \%$ CI. If conducting a meta-analysis will be a problem associated with substantial heterogeneity, the results will be presented using narrative synthesis.

\section{DISCUSSION}

Khat chewing is one of the most common serious public health concerns swaying the lives of people, particularly the most productive population. To halt this problem, a nationwide concerted evidence is largely essential. Therefore, this comprehensive systematic review and meta-analysis will bring well-summarised and concerted evidence.

\section{ETHICS AND DISSEMINATION}

The main intention of the study is to qualitatively describe earlier primary studies and pool the results of those studies, thus ethical clearance will not be required. The results of the study will be published in a reputable peer-reviewed journal and presented at different scientific research conferences. In addition, the results will be distributed to academic as well as other concerned institutions.

Contributors WGA conceived the research question, edited the protocol, and will review the articles, and extract and analyse the data. TAZ will participate in the search process, appraise the quality of the articles and extract the data. WWT will take part in the search process, and will analyse and interpret the results of the study. All authors read and approved this article prior to sending for publication.

Funding The authors have not declared a specific grant for this research from any funding agency in the public, commercial or not-for-profit sectors.

Competing interests None declared.

Patient consent Not required.

Provenance and peer review Not commissioned; externally peer reviewed.

Open access This is an open access article distributed in accordance with the Creative Commons Attribution Non Commercial (CC BY-NC 4.0) license, which permits others to distribute, remix, adapt, build upon this work non-commercially, and license their derivative works on different terms, provided the original work is properly cited, appropriate credit is given, any changes made indicated, and the use is non-commercial. See: http://creativecommons.org/licenses/by-nc/4.0/.

\section{REFERENCES}

1. Berhanu M, Aregash E, Alyi M. Socio-Economic impact of khat in Mana District, Jimma Zone, South Western Ethiopia. Discourse J Agric Food Sci 2014;2:21-32.

2. Reda AA, Moges A, Biadgilign S, et al. Prevalence and determinants of khat (Catha edulis) chewing among high school students in eastern Ethiopia: a cross-sectional study. PLoS One 2012;7:e33946.

3. Nencini P, Grassi MC, Botan AA, et al. Khat chewing spread to the somali community in Rome. Drug Alcohol Depend 1989;23:255-8.

4. Aseffa M. Socio-economic aspects of khat in the harrarghe administrative region. Antananarivo, Madagascar. Ethiopia: Proceedings of the International Conference on Khat, 1983.

5. Brenneisen R, Fisch HU, Koelbing U, et al. Amphetamine-like effects in humans of the khat alkaloid cathinone. Br J Clin Pharmacol 1990;30:825-8.

6. Cox G, Rampes H. Adverse effects of Khat chewing: a review. Journal of continuing professional development. Advances in Psychiatric Treatment 2003;9:456-63.

7. Gebissa E. Khat in the Horn of Africa: historical perspectives and current trends. J Ethnopharmacol 2010;132:607-14.

8. Kalix P. Catha edulis, a plant that has amphetamine effects. Pharm World Sci 1996;18:69-73.

9. Dhaifalah I, Santavý J. Khat habit and its health effect. a natural amphetamine. Biomed Pap Med Fac Univ Palacky Olomouc Czech Repub 2004;148:11-15. 
10. Zein ZA. Polydrug abuse among Ethiopian university students with particular reference to khat (Catha edulis). J Trop Med Hyg 1988;91:71-5.

11. Kennedy JG. The flower of paradise: the institutionalized use of the drug qat in North Yemen. D Reidel Publications: Dordrecht, 1987:98-100.

12. Kalix $P$, Braenden $O$. Pharmacological aspects of the chewing of khat leaves. Pharmacol Rev 1985;37:149-64.

13. Adam F, Hasselot $N$. [Khat: from traditional usage to risk of drug addiction]. Med Trop 1994;54:141-4.

14. Banjaw MY, Schmidt WJ. Behavioural sensitisation following repeated intermittent oral administration of Catha edulis in rats. Behav Brain Res 2005;156:181-9.

15. Banjaw MY, Schmidt WJ. Catha edulis extract and its active principle cathinone induce ipsilateral rotation in unilaterally lesioned rats. Behav Pharmacol 2006;17:615-20.

16. Giannini AJ, Miller NS, Turner CE. Treatment of khat addiction. $J$ Subst Abuse Treat 1992;9:379-82.

17. Kalix P. Khat: scientific knowledge and policy issues. $\mathrm{Br} J$ Addict 1987;82:47-53.

18. Elmai. Khat consumption and problems in somalia. Antananarivo, Madagascar: International Conference on Khat, 1983.

19. Morghemm M, Rufatm I. Cultivation and chewing of khat in the yemen arab republic. Antananarivo, Madagascar: International Conference on Khat, 1983.

20. Getajiu A, Krikoriaand N. The economic and social importance of khat and suggested research and services. Antananarivo, Madagascar: Proceedings of the International Conference on Khat, 1983.

21. Alhegami MA. Effects of Khat (cathaedulis) on some blood contents and the tissues of the digestive system of rabbits. Dissertation]Sana'a (Yemen: Sana'a University, 2001.

22. Singh BN. Newer concepts in the pathogenesis of Myocardial Ischemia: implications for evaluation of antianginal therapy Drugs. 1986;32:1-14.

23. Gough SP, Cookson I, Mayberry J, et al. Khat-induced schizophreniform psychosis in UK. Lancet 1984;1:455.

24. Giannini AJ, Castellani S. A manic-like psychosis due to khat (Catha edulis Forsk.). J Toxicol Clin Toxicol 1982;19:455-9.

25. Newton TF, Kalechstein AD, Duran S, et al. Methamphetamine abstinence syndrome: preliminary findings. Am J Addict 2004;13:248-55

26. Malaju MT, Asale GA. Association of Khat and alcohol use with HIV infection and age at first sexual initiation among youths visiting HIV testing and counseling centers in Gamo-Gofa Zone, South West Ethiopia. BMC Int Health Hum Rights 2013;13:10.

27. Kebede D, Alem A, Mitike G, et al. Khat and alcohol use and risky sex behaviour among in-school and out-of-school youth in Ethiopia. BMC Public Health 2005;5:109.

28. Alem A, Shibre T. Khat induced psychosis and its medico-legal implication: a case report. Ethiop Med J 1997;35:137-9.

29. Odenwald M, Neuner F, Schauer M, et al. Khat use as risk factor for psychotic disorders: a cross-sectional and case-control study in Somalia. BMC Med 2005;3:5.

30. Balint EE, Falkay G, Balint GA. Khat - a controversial plant. Wien Klin Wochenschr 2009;121:604-14.

31. Mwenda JM, Arimi MM, Kyama MC, et al. Effects of khat (Catha edulis) consumption on reproductive functions: a review. East Afr Med J 2003;80:318-23.

32. Yücel M, Lubman DI. Neurocognitive and neuroimaging evidence of behavioural dysregulation in human drug addiction: implications for diagnosis, treatment and prevention. Drug Alcohol Rev 2007;26:33-9.

33. Yücel M, Lubman DI, Harrison BJ, et al. A combined spectroscopic and functional MRI investigation of the dorsal anterior cingulate region in opiate addiction. Mol Psychiatry 2007;12:691-702.
34. Al-Motarreb A, Al-Habori M, Broadley KJ. Khat chewing cardiovascular diseases and other internal medical problems: the current situation and directions for future research. J Ethnopharmacol 2010;132:540-8.

35. Meressa K, Mossie A, Gelaw Y. Effect of substance use on the academic achievement of health officer and medical students of Jimma University, southwest Ethiopia. Ethiopian journal of health sciences 2009;19.

36. Kebede Y. Cigarette smoking and Khat chewing among college students in North West Ethiopia. Ethiopian Journal of Health Development 2002;16:9-17.

37. Basunaid S, van Dongen M, Cleophas TJ. Khat Abuse in Yemen: a population-based survey. Clin Res Regul Aff 2008;25:87-92.

38. Ageely HM. Prevalence of Khat chewing in college and secondary (high) school students of Jazan region, Saudi Arabia. Harm Reduct J 2009;6:11.

39. Alsanosy RM, Mahfouz MS, Gaffar AM. Khat chewing among students of higher education in Jazan region, Saudi Arabia: prevalence, pattern, and related factors. Biomed Res Int 2013;2013:1-7.

40. Samy SM, Khamis AK, Khalid MM, et al. Prevalence and predictors of khat chewing among students of Jazan University, Jazan, Kingdom of Saudi Arabia. Int $J$ of Preventive and Public Health Sciences 2017;2

41. Bizuayehu G, Netsanet $H$, Prevalence BW. Factors and consequences of khat chewing among high school students of Gondar Town, Northwestern Ethiopia. Pharmacologyonline 2009;3:387-97.

42. Astatkie A, Demissie M, Berhane $Y$, et al. Prevalence of and factors associated with regular khat chewing among university students in Ethiopia. Subst Abuse Rehabil 2015;6:41-50.

43. Lakew A, Tariku B, Deyessa N, et al. Prevalence of $<i>C$ Catha edulis $</ i>$ (Khat) Chewing and Its Associated Factors among Ataye Secondary School Students in Northern Shoa, Ethiopia. Adv Appl Sociol 2014;04:225-33.

44. Gebrehanna E, Berhane Y, Worku A. Khat chewing among Ethiopian University Students-a growing concern. BMC Public Health 2014;14:1198.

45. Adugna F, Jira C, Molla T. Khat chewing among Agaro secondary school students, Agaro, southwestern Ethiopia. Ethiop Med J 1994;32:161-6.

46. Laswar AK, Darwish $\mathrm{H}$. Prevalence of cigarette smoking and khat chewing among Aden university medical students and their relationship to BP and body mass index. Saudi J Kidney Dis Transpl 2009;20:862.

47. Deressa W, Azazh A. Substance use and its predictors among undergraduate medical students of Addis Ababa University in Ethiopia. BMC Public Health 2011;11:660.

48. Kassa A, Loha E, Esaiyas A. Prevalence of khat chewing and its effect on academic performance in Sidama zone, Southern Ethiopia. Afr Health Sci 2017;17:175-85.

49. Stroup DF, Berlin JA, Morton SC, et al. Meta-analysis of observational studies in epidemiology: a proposal for reporting. Meta-analysis Of Observational Studies in Epidemiology (MOOSE) group. JAMA 2000;283:2008-12.

50. Moher D, Shamseer L, Clarke M, et al. Preferred reporting items for systematic review and meta-analysis protocols (PRISMA-P) 2015 statement. Syst Rev 2015;4:1.

51. Aromataris E, Fernandez R, Godfrey CM, et al. Summarizing systematic reviews: methodological development, conduct and reporting of an umbrella review approach. Int J Evid Based Healthc 2015;13:132-40.

52. DerSimonian R, Laird N. Meta-analysis in clinical trials revisited. Contemp Clin Trials 2015;45:139-45.

53. Higgins JP, Thompson SG. Quantifying heterogeneity in a metaanalysis. Stat Med 2002;21:1539-58. 